\title{
The unsolved problem of otitis media in indigenous populations: A systematic review of upper respiratory and middle ear microbiota in indigenous children
}

1 Andrea Coleman, a.coleman2@uq.edu.au ${ }^{1,2^{*}}$

2 Amanda Wood, Amanda.Wood2@health.qld.gov.au ${ }^{3}$

3 Seweryn Bialasiewicz, seweryn@uq.edu.au ${ }^{2}$

4 Robert S Ware, r.ware@griffith.edu.au ${ }^{4}$

5 Robyn L Marsh, Robyn.Marsh@menzies.edu.au ${ }^{5}$

6 Anders Cervin, a.cervin@uq.edu.au,6

$7 \quad{ }^{1}$ Faculty of Medicine, The University of Queensland, Brisbane, Queensland, Australia

$8{ }^{2}$ Queensland Pediatric Infectious Disease Laboratory, Centre for Children's Health Research,

9 Children's Health Queensland Hospital \& Queensland University of Technology, Child Health

10 Research Centre, The University of Queensland, Brisbane, Queensland, Australia

$11{ }^{3}$ The Deadly Ears Program, Children's Health Queensland Hospital and Health Service,

12 Brisbane, Queensland, Australia

$13{ }^{4}$ Menzies Health Institute Queensland, Griffith University, Brisbane, Queensland, Australia

$14{ }^{5}$ Menzies School of Health Research, Charles Darwin University, Darwin, Northern Territory,

15 Australia 
$16{ }^{6}$ Department of Otolaryngology Head \& Neck Surgery, Royal Brisbane and Women's Hospital,

17 Brisbane, Queensland, Australia

$18 *$ Correspondence:

19 Dr Andrea Coleman

20 a.coleman2@uq.edu.au

21 Queensland Paediatric Infectious Disease Laboratory

22 Centre for Children's Health Research

2362 Graham Street, South Brisbane

24 QLD 4101, Australia 


\section{Abstract}

27 Background: Otitis media (OM) imposes a great burden of disease in indigenous populations

28 around the world, despite a variety of treatment and prevention programs. Improved

29 understanding of the pathogenesis of $\mathrm{OM}$ in indigenous populations is required to advance

30 treatment and reduce prevalence. We conducted a systematic review of the literature exploring

31 upper airway and middle ear microbiota in relation to $\mathrm{OM}$ in indigenous children.

32 Methods: Papers targeting microbiota in relation to $\mathrm{OM}$ in children $<18$ years indigenous to

33 Australia, New Zealand, North America, and Greenland were sought. MEDLINE, CINAHL,

34 EMBASE, Cochrane Library, and Informit databases were searched using key words. Two

35 independent reviewers screened titles, abstracts, and then full-text papers against inclusion

36 criteria according to PRISMA guidelines.

37 Results: Twenty-five papers considering indigenous Australian, Alaskan and Greenlandic

38 children were included. There were high rates of nasopharyngeal colonization with the three

39 main otopathogens (Haemophilus influenzae, Streptococcus pneumoniae, and Moraxella

40 catarrhalis) in indigenous children with OM. Middle ear samples had lower rates of otopathogen

41 detection, although detection rates increased when molecular methods were used. Pseudomonas

42 aeruginosa and Staphylococcus aureus were commonly detected in middle ear discharge of

43 children with chronic suppurative OM. There was significant heterogeneity between studies,

44 particularly in microbiological methods, which were largely limited to culture-based detection of

45 the main otopathogens.

46 Conclusions: There are high rates of otopathogen colonization in indigenous children with OM.

47 Chronic suppurative OM appears to be associated with a different microbial profile. Beyond the 
48 main otopathogens, the data are limited. Further research is required to explore the entire upper

49 respiratory tract/ middle ear microbiota in relation to OM, with the inclusion of healthy

50 indigenous peers as controls.

51 Keywords: otitis media, indigenous, microbiota, pediatrics, systematic review

52 


\section{Introduction}

54 Otitis media (OM) describes a spectrum of pathologies that involve inflammation and/or

55 infection in the middle ear. This spectrum encompasses a continuum from acute to chronic

56 disease that is clinically characterised by fluid in the middle ear [1-4]. OM is highly prevalent in

57 indigenous populations globally, particularly when compared to non-indigenous peers [5, 6], and

58 often occurs earlier, more frequently and in more severe forms [4, 5, 7]. Prevalence data reports

59 that up to one-third of Greenlandic and Alaskan Inuit, Native American and Australian

60 Indigenous children suffer from chronic suppurative OM (CSOM) [6, 8-11]. The World Health

61 Organization considers CSOM prevalence of $\geq 4 \%$ indicative of a public health problem serious

62 enough to require urgent attention [12]. OM-related complications result in approximately

6321,000 deaths each year worldwide [13]. OM-associated hearing loss can impact significantly on

64 language and social skills development, school attendance and educational outcomes, and

65 downstream effects such as greater contact with the criminal justice system later in life [4, 14,

66 15]. Medical interventions including liberal antibiotic prescription and vaccination programs

67 have limited effectiveness in indigenous populations [16-19], thus new treatment avenues need

68 to be considered.

69 The reasons for high OM prevalence in indigenous populations are likely to be multi-factorial.

70 Risk factors include poverty, inadequate housing, overcrowding, and exposure to environmental

71 tobacco smoke $[6,8,20,21]$. These risk factors are ubiquitous across indigenous populations

72 worldwide [22]. Genetic susceptibility to OM has not been studied in indigenous populations

$73[23,24]$.

74 The microbiota of the upper respiratory tract (URT) is an important OM risk factor across all

75 populations. Most research to date has focused on the role of the three main otopathogens: 
77 [25]. It is not currently clear whether commensal bacteria amongst the URT microbiota

78 contribute to, or mitigate, OM risk in indigenous children. In non-indigenous children, $16 \mathrm{~S}$

79 ribosomal RNA (rRNA) gene analyses have suggested that a 'healthy' nasopharyngeal (NP)

80 microbiota is more diverse than that of children with OM [26-29]. This 'healthy' NP microbiota

81 contains bacteria that may be protective or promote microbiota stabilization, including,

82 Moraxella, Corynebacterium, Dolosigranulum, Propionibacterium (Cutibacterium),

83 Lactococcus and Staphylococcus [26-29]. It is currently unknown whether these results are

84 generalizable to indigenous populations.

85 While high rates of OM are reported for many developing countries, indigenous populations, as

86 defined by the United Nations [30], share unique challenges in relation to OM. The aim of this

87 systematic review is to assess the data pertaining to the NP and middle ear microbiota in relation

88 to OM affecting indigenous children around the world.

\section{Methods}

90 Methods used for this systematic review were developed with reference to the Preferred

91 Reporting Items for Systematic Reviews and Meta-Analyses (PRISMA) Statement. The protocol

92 was registered with the International Prospective Register of Systematic Reviews (PROSPERO)

93 (CRD42016033905) prior to commencement.

\section{Inclusion criteria}

95 All studies exploring the microbiota of the URT (nose, nasopharynx, mouth, oropharynx, throat,

96 tonsils, adenoid, and middle ear) in relation to OM in indigenous children aged 0-18 years old

97 were included. For studies that included children without OM and/or did not report microbiology

98 results specifically for children with OM, either only middle ear data were included, or if only 
99 the NP was sampled, the studies were excluded. Indigenous populations from Australia, New

100 Zealand, Unites States of America, Canada, and Greenland were included.

\section{Search strategy}

102 Literature search strategies were developed in collaboration with a health sciences librarian using

103 medical subject headings (MeSH) and key words (Additional File 1). The following electronic

104 databases were searched from inception until 15 August 2017: MEDLINE (from 1946) and

105 CINHAL (from 1982) via EBSCOhost, EMBASE (from 1966), Cochrane Library (from 1996),

106 and Informit (from 1990- April). To ensure search saturation, we reviewed the reference lists of

107 relevant studies and sought unpublished clinical audits through the Australian Institute of Health

108 and Welfare (https://www.aihw.gov.au/) and The Australian Indigenous Health Info Net

109 (https://healthinfonet.ecu.edu.au/). Two independent reviewers (ACol and AW) revised titles and

110 abstracts, then full text publications with reference to the inclusion criteria. Study selection inter-

111 rater agreement between the two reviewers was calculated as the proportion of positive

112 agreement (PA) [31].

\section{Data extraction}

114 Two independent reviewers (ACol and AW) extracted data in duplicate onto a Microsoft Excel

115 spreadsheet. Publication authors were contacted where data had been represented graphically or

116 data were missing. We screened for multiple reports from the same study, and where multiple

117 reports existed, compared and extracted relevant data; if inconsistencies existed, we contacted

118 the authors for clarification. The following data were extracted for all studies meeting inclusion

119 criteria: publication year, geographical location, study design, number of participants, age range,

120 ethnicity, number of participants with an OM diagnosis, type of OM, number of controls,

121 anatomical location of sample(s), microbiota investigation method, type and quantity of bacteria, 
122 viruses, and fungi detected from each anatomic site. For the purpose of the review 'culture' is

123 defined as culture targeting the three main otopathogens and 'extended culture' is defined as

124 culture used to detect bacteria beyond these otopathogens. Only quantitative PCR (qPCR) data

125 were included when both culture and qPCR were used. For longitudinal studies, data relating to

126 both the number of swabs and number of children were extracted, when there were multiple

127 swabs per child. For data obtained from clinical trials, we included data only from samples

128 collected prior to randomization.

\section{Data analysis}

130 Where there were a sufficient number of studies, meta-analysis of proportions were calculated

131 using random effects analysis via Stata/IC 15, otherwise we synthesized the data into a

132 systematic narrative. We calculated heterogeneity using $\mathrm{I}^{2}$ statistic.

\section{Risk of bias assessment}

134 Two independent reviewers (ACol and AW) assessed the risk of bias for each study with

135 reference to the Critical Appraisal Skills Program (CASP) Cohort Study Checklist [32]. Within

136 the CASP Checklist, we assessed for the following confounding variables: age, overcrowding,

137 antibiotic use, daycare/ school attendance, and concurrent respiratory/ upper respiratory tract

138 infection. Study quality was categorized as 'poor', 'moderate' or 'good' based on the CASP

139 Checklist. The overall quality of evidence was judged as high, moderate, low, and very low [33].

\section{Results}

141 The initial search identified 5592 articles. After screening titles, the abstracts of 956 articles and

142332 full text publications were reviewed (Figure 1). There was substantial PA between the

143 reviewers of titles $(\mathrm{PA}=0.68)$ and abstracts $(\mathrm{PA}=0.79)$. Twenty-five articles met the inclusion

144 criteria; these were from Australian Indigenous $(n=22)$, Greenlandic $(n=2)$ and Alaskan Inuit 
$145(n=1)$. No papers reported OM otopathogens or microbiota in Native American or New Zealand

146 Maori children.

147 Risk of bias assessment

148 According to the CASP risk of bias assessment, most studies (80\%) were judged as either 'poor'

149 or 'moderate', largely due to confounding variables not being considered (Table 1). Recruitment

150 bias was difficult to assess, as recruitment processes were often poorly documented. Only one

151 study [34] included healthy indigenous controls and another three [21, 35, 36] included children

152 without OM enrolled in longitudinal studies. Within indigenous populations, participants were

153 recruited from limited geographical regions, making generalization beyond these regions

154 difficult. Overall, the quality of the literature was 'low'.

\section{Heterogeneity}

156 The literature was limited by methodological and statistical heterogeneity across the studies,

157 including heterogeneity in study design, participant age, OM diagnosis, and laboratory methods

158 (Table 2). Where there were sufficient data to calculate $\mathrm{I}^{2}$; most were $>70 \%$, indicating

159 moderate-high heterogeneity (Figure 2-4).

160 OM clinical definitions and diagnosis

161 OM definitions used by the studies are outlined in Additional File 2. Acute OM (AOM)

162 definitions were consistently based on otoscopy and tympanometry. OM with effusion (OME)

163 was diagnosed based on a type-B tympanogram in 5/8 studies; the remaining three studies [37-

164 39] reported data from intra-operative middle ear effusion (MEE) samples, without specifying

165 OME diagnostic criteria. CSOM definitions were heterogeneous and included otorrhoea for $>2$

166 weeks [40, 41], >6 weeks [42], and broad descriptive terms [39, 43]. Three studies did not

167 describe specific OM diagnostic criteria [44-46]. 
169 Methods used to assess URT and middle ear bacteriology varied across studies (Table 2). Most

170 studies (13/25) used culture conditions specific for detection of the main otopathogens. Nine

171 studies used extended culture to detect a wider range of bacteria. For the culture-based studies,

172 methodological details varied. Most culture-based studies (13/22) described the agar plates used

173 and growth conditions [17, 21, 34, 36-40, 42, 43, 47-49]; however, reporting of phenotypic

174 isolate identification tests varied. The remaining studies used non-specific terms or referred to

175 other papers [44-46, 50-55].

176 Three studies used only molecular methods: two used species-specific qPCR targeting the main

177 otopathogens or Alloiococcus otitidis [35, 56], and one used 16S rRNA gene sequencing [57].

178 One study used both culture and qPCR [48]. The three studies using qPCR [35, 48, 56] used the

179 same gene targets for S. pneumoniae and M. catarrhalis. Two studies used the hpd gene to detect

$180 H$. influenzae $[35,56]$ while another used an alternative gene target, $h p d 3$ [48]. Only one paper

181 used qPCR to detect $A$. otitidis [56].

\section{Bacteriology}

183 Acute otitis media

184 AOM bacteriology was reported for Australian and Greenlandic indigenous children, with high

185 prevalence of the three main otopathogens in NP/nose and middle ear specimens across both

186 populations (Figure 2 and Additional File 3). Co-infection with >1 otopathogen was common in

187 the NP, although less frequent in MED (Additional File 3). NP colonization by S. pneumoniae

188 (both populations) or M. catarrhalis (Australian Indigenous) was significantly related to AOM

189 when compared to indigenous peers without OM [34, 35]. Beyond the main otopathogens, $A$.

190 otitidis, Staphylococcus spp. and $\beta$ hemolytic streptococcus were also detected in the middle ear 
191 discharge (MED) of children with AOM with perforated tympanic membrane (AOMwP)

192 (Additional File 4).

193 Otitis media with effusion

194 The one study investigating NP microbiota, and all but one study exploring MEE in children

195 with OME were from Australian Indigenous children. The three main otopathogens were highly

196 prevalent in the NP in children with OME (Figure 3 and Additional File 3), although only $S$.

197 pneumoniae and M. catarrhalis were significantly related to OME in the one study that included

198 a control group [35]. Culture-based studies reported a low prevalence of otopathogens in MEE

199 (Figure 3, Additional File 3); however, much higher rates were detected in the single study that

200 used molecular methods [57] (Figure 3). Other bacteria detected in MEE by extended culture

201 included A. otitidis, Corynebacterium spp., Pseudomonas aeruginosa and S. aureus (Additional

202 File 4). The single 16S rRNA gene sequencing analysis (Australian Indigenous children) [57]

203 found high rates of the genera Dolosigranulum, Moraxella, Haemophilus and Streptococcus

204 (Mitis group) in the NP, and Alloiococcus, Haemophilus and Corynebacterium in MEE

205 (Additional File 5)

206 Chronic suppurative otitis media

207 All but one study investigating CSOM were from Australian Indigenous children. The most

208 commonly reported bacteria from culture-based studies of MED from children with CSOM were

209 P. aeruginosa, S. aureus, and H. influenzae (Figure 4). P. aeruginosa and H. influenzae were

210 often detected in Australian Indigenous children, but not in the single study of Greenlandic Inuit

211 children (Figure 4). Yeasts were reported in two Australian Indigenous studies (Additional File

212 4); one study [40] only detected Candida, Aspergillus, Fusarium, Alternaria, Rhodotorula,

213 Auerobasidium or Acrinomium in 5\% of MED samples. The other study [43] did not identify or 
214 specify the yeasts or fungi detected. No study used molecular methods to explore the URT or

215 middle ear microbiota in CSOM.

216 Nasopharyngeal carriage as a risk factor for otitis media

217 Two prospective cohort studies in Australian Indigenous children explored NP carriage of the

218 three main otopathogens as a risk factor for OM (all types) [21, 36]. A birth cohort study by

219 Leach et al. found that 31/36 (86\%) children with their first episode of OM were colonized with

220 at least one otopathogen [21]. This relationship between NP colonization and OM was stronger

221 when $>1$ otopathogen was detected in the $\mathrm{NP}$ (odds ratio (OR) $=33.6,95 \%$ CI 7.9 to 144) [21].

222 More recently, Sun et al. found that in Australian Indigenous children, early colonization (1 to

$223<3$ months of age) with $H$. influenzae was associated with $\mathrm{OM}$ in the first two years of life $(\mathrm{OR}=$

$2243.71,95 \%$ CI 1.22 to 11.23) [36]. All children (100\%) who carried H. influenzae with either of

225 the other main otopathogens were subsequently diagnosed with OM [36].

\section{Virology}

227 One Australian [35] and one Greenlandic study [34] tested for viruses in children with OM

228 (Additional File 4). These studies used different methods for viral detection and, aside from

229 rhinovirus, tested for different viruses (Table 2). In Indigenous Australian children, only

230 adenovirus in the NP was related to AOM (19\%) and AOMwP (20\%) compared to control

231 children (6\%) [35]. There was no relationship between the detection of viruses in the NP and

232 OME [35]. In Greenlandic Inuit children, enteroviruses, rhinoviruses or 'unspecified virus' in the

233 NP was related to AOM, compared to controls [34]. No studies tested for viruses in middle ear

234 specimens in AOMwP, OME, or CSOM.

\section{Biofilm}


236 One Greenlandic study used PNA-FISH to test for biofilm in middle ear specimens from children

237 with CSOM or OME (obtained via sterile aspiration) [39]. Biofilm was detected in 5/6 (83\%)

238 MED samples from children with CSOM using a Eubacterial probe, but not in MEE from seven

239 children with OME [39]. Further testing with species-specific probes found most biofilms $(66 \%)$

240 contained S. aureus. One further sample contained a Stenotrophomonas maltophilia biofilm. For

241 S. aureus and S. maltophilia, there was a 100\% agreement between culture, Gram-staining and

242 PNA-FISH results [39]. Species-specific probes targeting the main otopathogens were not tested.

\section{Discussion}

244 This systematic review found the NP of most indigenous children with OM were colonized with

245 the main otopathogens, particularly those with AOM. In contrast, children with CSOM

246 demonstrate a different middle ear microbial profile compared to children with AOM and OME.

247 Beyond the typical culturable bacteria, data are sparse, limiting our understanding of how the

248 broader microbiota of the URT may contribute to OM pathogenesis and persistence in

249 indigenous populations. The entire OM microbiome across all indigenous populations needs

250 further investigation.

251 Our analysis highlights the important role of S. pneumoniae and H. influenzae in the

252 pathogenesis of $\mathrm{AOM} / \mathrm{AOMwP}$ and $\mathrm{OME}$ across indigenous populations, consistent with data

253 from non-indigenous populations [58]. These otopathogens were detected at low rates in middle

254 ear samples from children with AOMwP and OME; however, when molecular techniques were

255 employed detection rates were much higher, particularly for $H$. influenzae [57], consistent with

256 the increased sensitivity of molecular methods compared to culture $[56,59]$. This suggests that

257 current data, which are predominantly culture-based, may underestimate the prevalence of

258 otopathogen colonisation in middle ear samples from indigenous children. 
A different pathogen profile was reported from children with CSOM, including, P. aeruginosa,

260 S. aureus, H. influenzae and fungi/ yeasts. Commensurate with this result, culture-based

261 literature from non-indigenous children with CSOM often report P. aeruginosa and S. aureus in

262 MED [60-65]. 16S rRNA gene sequencing of MED from children and adults with CSOM in New

263 Zealand further detected Alloiococcus and Streptococcus [66]. In the CSOM studies included in

264 this review, Alloiococcus would not have been detected, if present, as the specialist culture

265 conditions or PCR required to detect this species were not used. The chronic perforation of the

266 tympanic membrane in CSOM may allow for secondary infection of the middle ear by microbes

267 present in the external auditory canal and could account for the different microbial profile

268 compared to other types of OM. Confirming this; however, is difficult, particularly where a child

269 has had prolonged otorrhoea with ear discharge draining into the canal. Sampling the canal flora

270 of children with intact tympanic membranes as a comparison, may provide a solution.

271 Biofilms have been reported in middle ear specimens from non-indigenous children with CSOM

272 and OME [41, 59, 67-69]; however, this systematic review uncovered very little data pertaining

273 to biofilm in relation to $\mathrm{OM}$ in indigenous children. Considering the high rates of chronic OM,

274 particularly CSOM, this is a noteworthy deficit of the literature.

275 This systematic review suggests other microbes, beyond the main otopathogens, may be

276 contributing to $\mathrm{OM}$ in indigenous populations; however, there are few data relating to these taxa.

277 Furthermore, detection of these microbes can require specific laboratory techniques. For

278 example, A. otitidis detection requires extended culture methods [37] or molecular methods [56,

279 57]. Where these methods have been used, A. otitidis was commonly detected [37, 56, 57];

280 however, it remains controversial whether detection of this species is associated with the middle

281 ear infection or specimen contamination by canal flora [56]. Viruses were seldom investigated in 
282 the included studies, and when investigated, different viruses were sought, and detection

283 methods varied. Furthermore, only NP specimens were tested. Viruses are likely to play an

284 important role in OM pathogenesis [70], through numerous potential mechanisms including

285 altering the host immune response [71] and reducing response to antibiotic therapy [72]. Further

286 research is required to determine the contribution of respiratory viruses in OM pathogenesis.

\section{Limitations of the current literature}

288 The current literature is limited by methodological heterogeneity, in both the types of laboratory

289 methods used and the OM definitions and diagnoses. The greatest source of methodological

290 heterogeneity was the diversity of methods used to analyze the samples with varying specificities

291 and sensitivities. Inconsistencies in OM definitions and diagnoses were most apparent in the

292 CSOM data, reflecting the absence of internationally accepted definitions [73]. Other OM

293 diagnoses were more consistent, largely because the data was published from a limited number

294 of research groups. International guidelines on OM definitions, diagnosis and investigation of

295 URT/ middle ear microbiota are needed. This will allow for more meaningful comparison of

296 studies from around the world and facilitate future meta-analysis.

297 The quality of the data included in this review is impacted by the absence of healthy indigenous

298 controls; limited information on participant recruitment; poor consideration of confounding

299 variables; multiple studies where the microbiology is not the primary aim of the study; and

300 population overlap. The absence of healthy indigenous control children may reflect the high

301 burden of disease in many of these populations, for example $<10 \%$ of Australian Indigenous

302 children living in remote areas have healthy ears [7]. To establish a 'OM microbiota',

303 comparison with healthy indigenous peers is required. Similarly, if samples from the external

304 auditory canal are included when analyzing middle ear specimens, we may be able to delineate 
305 the role of microbes as contaminate, pathogen or secondary pathogen (e.g. A. otitidis). There

306 was significant population overlap and small geographical area of recruitment for many studies

307 in Australian Indigenous children. There is documented discordance in OM burden and

308 prevalence of otopathogen colonization between urban and remote Australian Indigenous

309 children $[74,75]$. Therefore, this limited area of recruitment may impact on generalization of

310 results across Australian Indigenous children.

\section{$311 \quad$ Future directions}

312 To further our understanding of OM pathogenesis in indigenous populations, and to build upon

313 the current pathogen-based disease model, further research is required to investigate the vast

314 array of microbes that can occupy the URT, and how they relate to the known otopathogens to

315 cause disease. The inclusion of healthy indigenous peers is vital to this goal. Identification of a

316 'healthy' microbiota in indigenous populations may uncover 'protective' microbes that can be

317 developed into microbiome/ probiotic therapies to protect children from OM. To achieve this

318 outcome, next generation sequencing can enable deeper exploration of the microbiota without $a$

319 priori assumptions about the underlying bacterial community, which is required to guide culture-

320 based methods. 16S rRNA gene sequencing, although limited by poor resolution at the species-

321 level, can be augmented by qPCR to provide species-level identification [76]; however, this

322 requires a priori assumptions about the bacteria that should be targeted. Likewise, qPCR for

323 specific viruses is limited by a priori assumptions. These limitations may be overcome with

324 metagenomic shotgun sequencing if the method can be optimized to overcome the technical

325 limitations related to high proportions of human DNA in middle ear specimens. Alternatively,

326 extended culture with MALDI-TOF can be used to provide a broader analysis of the microbiota 
327 to the species level [77] and has the benefit of providing material for further studies, such as

328 bacterial interference studies.

\section{Conclusions}

330 The URT microbiology in OM is highly complex and dynamic. Through this systematic review

331 we demonstrated that the three main otopathogens are important in the pathogenesis of AOM

332 across the indigenous populations included, and in non-indigenous peers. There is; however, a

333 vast community of microbes present in the URT. How these microbes interact to promote or,

334 perhaps more importantly protect, indigenous children from OM requires further investigation.

335 With a more wholistic understanding of the microbial pathogenesis of OM in indigenous

336 populations, we will be better equipped to develop new methods to prevent and treat OM in these

337 populations.

\section{Competing interests}

339 The authors declare that they have no competing interests.

\section{$340 \quad$ Funding}

341 Coleman received support from an Avant Doctors in Training Research Scholarship, an NHMRC

342 Postgraduate Research Scholarship (APP1133366) and a Queensland Health Junior Doctor

343 Fellowship.

344 Marsh is supported by the NHMRC CRE in Respiratory Health of Aboriginal and Torres Strait

345 Islander Children, grant number 1040830.

346 Cervin is support by the University of Queensland Faculty of Medicine Strategic Funding and

347 The Garnett Passe \& Rodney Williams Memorial Foundation. 


\section{References}

349 1. Coticchia JM, Chen M, Sachdeva L, Mutchnick S. New paradigms in the pathogenesis of otitis media in children. Front Pediatr. 2013;1:52.

2. Darwin Otitis Guidelines Group. Recommendations for Clinical Care Guidelines on the Management of Otitis Media in Aboriginal and Torres Strait Islander Populations. 2010.

3. Burns J, Thomson N. Review of ear health and hearing among Indigenous Australians. 2013. [http://www.healthinfonet.ecu.edu.au/other-health-conditions/ear/reviews/ourreview]. Accessed 02.03.2014.

4. Kong K, Coates HLC. Natural history, definitions, risk factors and burden of otitis media. Med J Aust. 2009;191:S39-S43.

5. Daly KA, Hoffman HJ, Jorunn Kvaerner K, Kvestad E, Casselbrant ML, Homoe P, Rovers MM. Epidemiology, natural history, and risk factors: Panel report from the Ninth International Research Conference on Otitis Media. Int J Pediatr Otorhinolaryngol. 2010;74:231-240.

6. Bhutta MF. Evolution and Otitis Media: A Review, and a Model to Explain High Prevalence in Indigenous Populations. Hum Biol. 2015;87:92-108.

7. Morris P, Leach AJ, Silberberg P, Mellon G, Wilson C, Hamilton E, Beissbarth J. Otitis media in young Aboriginal children from remote communities in Northern and Central Australia: a cross-sectional survey. BMC Pediatr. 2005;5:27.

8. Jervis-Bardy J, Sanchez L, Carney AS. Otitis media in Indigenous Australian children: review of epidemiology and risk factors. The Journal Of Laryngology And Otology. 2014;128 Suppl 1:S16-S27.

9. Baxter JD. Otitis media in Inuit children in the Eastern Canadian Arctic_-an overview1968 to date. Int J Pediatr Otorhinolaryngol. 1999;49:S165-S168.

10. Maynard JE, Fleshman JK, Tschopp CF. Otitis media in Alaskan Eskimo children: Prospective evaulation of chemoprophylaxis. JAMA. 1972;219:597-599.

11. Homøe P, Christensen RB, Bretlau P. Prevalence of otitis media in a survey of 591 unselected Greenlandic children. Int J Pediatr Otorhinolaryngol. 1996;36:215-230.

12. World Health Organisation. Prevention of hearing impairment from chronic otitis media. 2000.

13. Monasta L, Ronfan L, Marchetti F, Monico M, Vecchi Brumatti L, Bavcar A, Grasso D, Barbiero C, Tamburlini G. Burden of disease caused by otitis media: systematic review and global estimates. PloS ONE. 2012;7:e36226.

14. Williams CJ, Jacobs AM. The impact of otitis media on cognitive and educational outcomes. Med J Aust. 2009;191:S69-S72.

15. Closing the Gap Clearinghouse (AIHW \& AIFS). Ear disease in Aboriginal and Torres Strait Islander children. Resource sheet no. 35. Produced by the Closing the Gap Clearinghouse. 2014.

16. Mackenzie GA, Carapetis JR, Leach AJ, Morris PS. Pneumococcal vaccination and otitis media in Australian Aboriginal infants: comparison of two birth cohorts before and after introduction of vaccination. BMC Pediatrics. 2009;9:14-14.

17. Leach AJ, Wigger C, Beissbarth J, Woltring D, Andrews R, Chatfield M, Smith-Vaughan $\mathrm{H}$, Morris P. General health, otitis media, nasopharyngeal carriage and middle ear microbiology in Northern Territory Aboriginal children vaccinated during consecutive periods of 10-valent or 13-valent pneumococcal conjugate vaccines. Int J Pediatr Otorhinolaryngol. 2016;86:224-232. 
18. Nicholls TR, Leach AJ, Morris PS. The short-term impact of each primary dose of pneumococcal conjugate carriage: A systematic review and meta-analysis of randomised controlled trials. Vaccine. 2016;34:703-713.

19. Leach AJ, Morris PS. Antibiotics for the prevention of acute and chronic suppurative otitis media in children (Review). Evid Based Child Health. 2007;2:697-760.

20. Gunasekera H, Haysom L, Morris P, Craig J. The global burden of childhood otitis media and hearing impairment: A systematic review. Pediatrics. 2008;121:S107.

21. Leach AJ, Boswell JB, Asche V, Nienhuys TG, Matthews JD. Bacterial colonisation of the nasopharynx predicts very early onset and persistence of otitis media in Australian Aboriginal infants. Pediatr Infect Dis J. 1994;13:983-989.

22. World Health Organisation. Chronic suppurative otitis media: Burden of illness and management options. 2004.

23. Daly KA, Pirie PL, Rhodes KL, Hunter LL, Davey CS. Early Otitis Media Among Minnesota American Indians: The Little Ears Study. Am J Public Health. 2007;97:317322.

24. Koch A, Homøe P, Pipper C, Thomas H, Melbye M. Chronic suppurative otitis media in a birth cohort of children in Greenland: Population-based study of incidence and risk factors. Pediatr Infect Dis J. 2011;30:25-29.

25. Vergison A. Microbiology of otitis media: A moving target. Vaccine. 2008;26:G5-G10.

26. Biesbroek G, Tsivtsivadze E, Sanders E, Montijn R, Veenhoven R, Keijer BJF, Bogaert D. Early respiratory microbiota composition determines bacterial succession patterns and respiratory health in children. Am J Respir Crit Care Med. 2014;190:1283-1292.

27. Sakwinska O, Bastic Schmid V, Berger B, Bruttin A, Keitel K, Legpage M, Moine D, Ngom Bru C, Brüssow H, Gervaix A. Nasopharyngeal microbiota in healthy children and pneumonia patients. J Clin Microbiol. 2014;52:1590-1594.

28. Pettigrew M, Laufer A, Gent J, Kong Y, Fennie K, Metlay J. Upper respiratory tract microbial communities, acute otitis media pathogens, and antibiotic use in healthy and sick children. Appl Environ Microbiol. 2012;78:6262-6270.

29. Laufer AS, Metlay JP, Gent JF, Fennie KP, Kong Y, Pettigrew MM. Microbial communities of the upper respiratory tract and otitis media in children. Mbio. 2011;2:e00245-e00210.

30. Asia Pacific Forum, United Nations Human Rights Office of the High Commissioner. The United Nations Declaration on the Rights of Indigenous Peoples: A Manual for the National Rights of Insitutions. 2013.

31. de Vet HCW, Dikmans RE, Eekhout I. Specific agreement on dichotomous outcomes can be calculated for more than two raters. J Clin Epidemiol. 2017;83:85-89.

32. Critical Appraisal Skills Program. CASP Cohort Study Checklist. 2017. [http://www.casp-uk.net/casp-tools-checklists]. Accessed 2nd May 2017.

33. GRADE Working Group. Grading quality of evidence and strength of recommendations. BMJ. 2004;328:1490.

34. Homøe P, Prag J, Farholt S, Henrichsen J, Hornsleth A, Killian M, Skov Jensen J. High rate of nasopharyngeal carriage of potential pathogens among children in Greenland: Results of a clinical survey of middle-ear disease. Clin Infect Dis. 1996;23:1081-1090.

35. Binks MJ, Cheng AC, Smith-Vaughan H, Sloots T, Nissen M, Whiley D, McDonnell J, Leach AJ. Viral-bacterial co-infection in australian indigenous children with acute otitis media. BMC Infectious Diseases. 2011;11. 
36. Sun W, Jacoby P, Riley T, Bowman J, Leach AJ, Coates HLC, Weeks S, Cripps A, Lehmann D. Association between early bacterial carriage and otitis media in Aboriginal and non-Aboriginal children in a semi-arid area of Western Australia: a cohort study. BMC Infectious Diseases. 2012;12:366.

37. Ashhurst-Smith C, Hall S, Walker P, Stuart J, Hansbro P, Blackwell C. Isolation of Alloiococcus otitidis from Indigenous and non-Indigenous Australian children with chronic otitis media with effusion. FEMS Immunol Med Microbiol. 2007;51:163-170.

38. Parkinson A, Cuthbert M, Bulkow L, Davidson M, Petersen K, Clift S. Bacterial pathogens in chronic otitis media with effusion in Alaska Native children. Alaska Med. 1999;42:27-33.

39. Homøe P, Bjarnsholt T, Wessman M, Sørensen H, Johansen H. Morphological evidence of biofilm formation in Greenlanders with chronic suppurative otitis media. Eur Arch Otorhinolaryngol. 2009;266:1533-1538.

40. Couzos S, Lea T, Mueller R, Murray R, Culbong M. Effectiveness of ototopical antibiotics for chronic suppurative otitis media in Aboriginal children: a communitybased, multicentre, double-blind controlled trial. Med J Aust. 2003;179:185-190.

41. Jensen R, Johansen H, Bjarnsholt T, Eickhardt-Sørensen S, Homøe P. Recurrent otorrhea in chronic suppurative otitis media: is biofilm the missing link? Eur Arch Otorhinolaryngol. 2017;274:2741-2747.

42. Stephen A, Leach AJ, Morris PS. Impact of swimming on chronic suppurative otitis media in Aboriginal children: a randomised controlled trial. Med J Aust. 2013;199:51-55.

43. Leach AJ, Wood Y, Gadil E, Stubbs E, Morris PS. Topical Ciprofloxin versus topical Framycetin-Gramicidin-Dexamethasone in Australian Aboriginal children with recently treated chronic suppurative otitis media. Pediatr Infect Dis J. 2008;27:692-698.

44. Copeman R, Pashen D, Burger G. The health of the Aboriginal children of Cunnamulla, Western Queensland. Med J Aust. 1975;1:8-13.

45. Stuart J. Bacterial flora of the ear, nose, and throat in Aboriginal infants from Brisbane and Cherbourg. Med J Aust. 1975;1:10-44.

46. Stuart J, Quayle C, Lewis A, Harper J. Health, hearing and ear disease in Aboriginal school children. Med J Aust. 1972;22:855-859.

47. Leach AJ, Wigger C, Hare K, Hampton V, Beissbarth J, Andrews R, Chatfield M, SmithVaughan H, Morris PS. Reduced middle ear infection with non-typeable Haemophilus influenzae, but not Streptococcus pneumoniae, after transition to 10-valent pneumococcal non-typeable H. influenzae protein D conjugate vaccine. BMC Pediatr. 2015;15.

48. Smith-Vaughan HC, Binks MJ, Marsh RL, Kaestli M, Ward L, Hare KM, Pizzutto SJ, Thornton RB, Morris PS, Leach AJ. Dominance of Haemophilus influenzae in ear discharge from Indigenous Australian children with acute otitis media with tympanic membrane perforation. BMC Ear, Nose and Throat Disord. 2013;13.

49. Stuart J, Butt H, Walker P. The microbiology of glue ear in Australian Aboriginal children. Journal Of Paediatrics And Child Health. 2003;39:665-667.

50. Gibney KB, Morris PS, Carapetis JR, Skull SA, Smith-Vaughan HC, Stubbs E, Leach AJ. The clinical course of acute otitis media in high-risk Australian Aboriginal children: a longitudinal study. BMC Pediatr. 2005;5:16-16.

51. Leach A, MacKenzie G, Hare KM, Stubbs E, Beissbarth J, Kennedy M, Wilson C, Mellon G, Wigger C, Tippakalipa P, et al. Microbiology of acute otitis media with perforation (AOMwiP) in Aboriginal children living in remote communities - monitoring 
the impact of 7-valent pneumococcal conjugate vaccine (7vPCV). Int Congr Ser. 2006;1289:89-92.

52. MacKenzie GA, Carapetis JR, Leach AJ, Morris PS. Pneumococcal vaccination and otitis media in Australian Aboriginal infants: comparison of two birth cohorts before and after introduction of vaccination. BMC Pediatr. 2009;9.

53. Morris PS, Gadil G, McCallum G, Wilson C, Smith-Vaughan H, Torzillo P, Leach AJ. Single-dose azithromycin versus seven days of amoxycillin in the treatment of acute otitis media in Aboriginal children (AATAAC): a double blind, randomised controlled trial. Med J Aust. 2010;192:24-29.

54. Leach AJ, Morris PS, Mathews J, Chronic Otitis Media Intervention Trial - One (COMIT1) group. Compared to placebo, long-term antibiotics resolve otitis media with effusion (OME) and prevent acute otitis media with perforation (AOMwiP) in a high-risk population: a randomized controlled trial. BMC Pediatr. 2008;8.

55. Dawson VM, Coelen RJ, Murphy S, Graham D, Dyer H, Sunderman J. Microbiology of chronic otitis media with effusion among Australian Aboriginal children: role of Chlamydia trachomatis. The Australian Journal Of Experimental Biology And Medical Science. 1985;63:99-107.

56. Marsh RL, Binks MJ, Beissbarth J, Christensen P, Morris PS, Leach AJ, Smith-Vaughan H. Quantitative PCR of ear discharge from Indigenous Australian children with acute otitis media with perforation supports a role for Alloiococcus otitidis as a secondary pathogen. BMC Ear Nose Throat Disord. 2012;12.

57. Jervis-Bardy J, Rogers GB, Morris PS, Smith-Vaughan HC, Nosworthy E, Leong LEX, Smith RJ, Weyrich LS, De Haan J, Carney AS, et al. The microbiome of otitis media with effusion in Indigenous Australian children. Int J Pediatr Otorhinolaryngol. 2015;79:1548-1555.

58. Ngo CC, Massa HM, Thornton RB, Cripps AW. Predominant bacteria detected from the middle ear fluid of children experiencing otitis media: A systematic review. PLoS ONE. 2016;11:e0150949.

59. Hall-Stoodley L, Gieseke A, Nistico L, Nguyen D, Hayes J, Forbes M, Greenberg DP, Dice B, Burrows A, Wackym A, et al. Direct detection of bacterial biofilms on the middle-ear mucosa of children with chronic otitis media. JAMA. 2006;296:202-211.

60. Prakash R, Juyal D, Negi V, Pal S, Adekhadi S, Sharma M, Sharma N. Microbiology of chronic suppurative otitis media in a tertiary care setup of Uttarakhand State, India. N Am J Med Sci. 2013;5:282-287.

61. Kumar R, Agarwal R, Gupta S. A microbiological study of chronic suppurative otitis media. Int J Recent Sci Res. 2015;6:5487-5490.

62. Yeo S, Park D, Hong S, Cha C, Kim M. Bacteriology of chronic suppurative otitis media-a multicenter study. Acta Otolaryncol. 2007;127:1062-1067.

63. Dayasena R, Dayasiri M, Jayasuriya C, Perera D. Aetiological agents in chronic suppurative otitis media in Sri Lanka. Australas Med J. 2011;4:101-104.

64. Aflobi O, Salaudeen A, Ologe F, Nsabuisi C, Nwawolo. Pattern of bacterial isolates in the middle ear discharge of patients with chronic suppurative otitis media in a tertiary hospital in North central Nigeria. Afr Health Sci. 2012;12:362-367.

65. Mittal R, Lisi C, Gerring R, Mittal J, Mattal J, Mathee K, Narasimhan G, Azad R, Grati M, Eshranghi A, et al. Current concepts in the pathogenesis and treatment of chronic suppurative otitis media. J Med Microbiol. 2015;64:1103-1116. 
66. Neef M, Biswas K, Hoggard M, Taylor M, Douglas R. Molecular microbiological profile of chronic suppurative otitis media. J Clin Microbiol. 2016;54:2538-2546.

67. Kaya E, Dag I, Incesulu A, Gurbuz MK, Acar M, Birdane L. Investigation of the presence of biofilms in chronic suppurative otitis media, nonsuppurative otitis media, and chronic otitis media with cholesteatoma by scanning electron microscopy. The Scientific World Journal. 2013;2013:638715.

68. Lee MR, Pawlowski KS, Luong A, Furze AD, Sargent Roland P. Biofilm presence in humans with chronic suppurative otitis media. Otolaryngol Head Neck Surg. 2009; 141:567-571.

69. Thornton RB, Rigby PJ, Wiertsema SP, Filion P, Langlands J, Coates H, Vijayasekaran S, Keil AD, Richmond PC. Multi-species bacterial biofilm and intracellular infection in otitis media. BMC Pediatr. 2011;11:94.

70. Heikkinen T, Chonmaitree T. Importance of respiratory viruses in acute otitis media. Clin Microbiol Rev. 2003;16:230-241.

71. Abramson J, Giebink G, Quie P. Influenza A virus-induced polymorphonuclear leukocyte dysfunction in the pathogenesis of experimental pneumococcal otitis media. Infect Immun. 1982;36:289-296.

72. Chonmaitree T, Owen M, Howie V. Respiratory viruses interfere with bacteriologic response to antibiotic in children with acute otitis media. J Infect Dis. 1990;162:546-549.

73. Homøe P, Kvarner K, Damoiseaux RAMJ, van Dongen TMA, Gunasekera H, Jensen R, Morris P, Weinreich HM. Panel 1: Epidemiology and diagnosis. Otolaryngol Head Neck Surg. 2017;156:S1-S21.

74. Gunasekera H, Morris PS, Daniels J, Couzos S, Craig JC. Otitis media in Aboriginal children: the discordance between burden of illness and access to services in rural/remote and urban Australia. J Paediatr Child Health. 2009;45:425-430.

75. O'Grady K, Hall K, Sloots T, Anderson J, Chang A. Upper airway viruses and bacteria in urban Aboriginal and Torres Strait Islander children in Brisbane: A cross-sectional study. BMC Infectious Diseases. 2017;17:245.

76. Sillanpää S, Kramna L, Oikarinen S, Sipilä M, Rautiainen M, Aittoniemi J, Laranne J, Hyöty H, Cinek O. Next-generation sequencing combined with specific PCR assays to determine the bacterial 16S rRNA gene profiles of middle ear fluid collected from children with acute otitis media. mSphere. 2017;2:e00006-00017.

77. Sung JY, Hwang Y, Shin HM, Park MS, Lee SH, Yong D, Lee K. Untility of cenventional culture and MALDI-TOF MS for indentification of microbial communities in brochoalveolar lavage fluid in comparison with GS junior next generation sequencing system. Ann Lab Med. 2018;38:110-118. 


\begin{tabular}{|c|c|c|c|c|c|c|c|c|c|c|c|}
\hline & 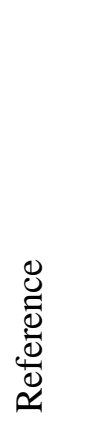 & 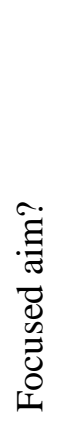 & 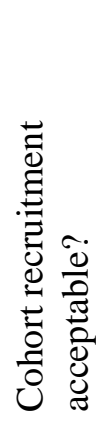 & 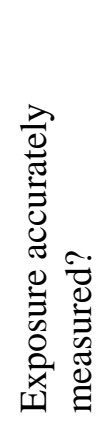 & 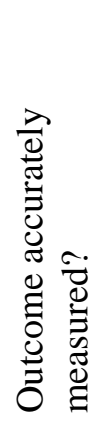 & 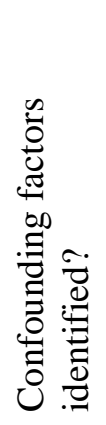 & 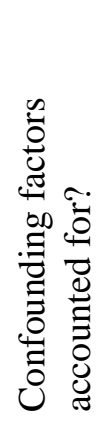 & 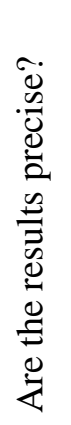 & 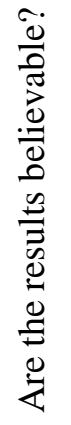 & 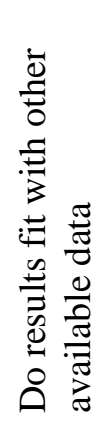 & 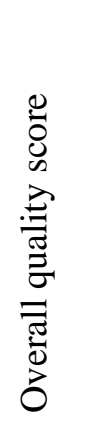 \\
\hline 1972, Stuart* & [46] & + & + & - & - & + & - & - & - & - & Poor \\
\hline 1975, Copeman & [44] & - & - & - & - & - & - & $?$ & - & - & Poor \\
\hline 1975, Stuart & [45] & + & + & - & - & - & - & - & - & - & Poor \\
\hline 1985, Dawson & [55] & + & - & + & + & - & - & $?$ & - & - & Poor \\
\hline 1994, Leach & [21] & + & + & + & + & - & - & - & + & + & Mod \\
\hline 1996, Homøe & [34] & + & + & + & + & + & + & + & + & + & Good \\
\hline 1999, Parkinson & {$[38]$} & + & + & + & + & - & - & + & + & + & Mod \\
\hline 2003, Couzos* & [40] & + & $?$ & + & + & + & + & + & + & + & Good \\
\hline 2003, Stuart & [49] & + & $?$ & + & + & + & - & + & + & + & Mod \\
\hline 2005,Gibney & [50] & + & + & + & $?$ & - & - & + & + & + & Poor \\
\hline 2006, Leach* & [51] & + & $?$ & + & + & - & - & - & - & + & Poor \\
\hline $\begin{array}{l}\text { 2007, Ashhurst- } \\
\text { Smith }\end{array}$ & {$[37]$} & + & $?$ & + & + & - & - & $?$ & + & - & Mod \\
\hline 2008, Leach* & [43] & + & + & + & + & - & - & + & + & + & Mod \\
\hline 2008, Leach* & [54] & + & + & + & + & - & - & + & + & + & Poor \\
\hline 2009, Homøe* & [39] & + & + & + & + & - & - & - & - & - & Poor \\
\hline $\begin{array}{l}2009, \\
\text { Mackenzie* }\end{array}$ & [52] & + & $?$ & - & + & - & - & + & - & + & Poor \\
\hline 2010, Morris $*$ & [53] & + & + & + & + & + & + & + & + & + & Good \\
\hline 2011, Binks & [35] & + & $?$ & $?$ & + & - & - & + & + & + & Poor \\
\hline 2012, Marsh & [56] & + & $?$ & + & + & - & - & + & + & + & Mod \\
\hline 2012, Sun & [36] & + & + & + & + & + & + & + & + & + & Good \\
\hline $\begin{array}{l}\text { 2013, Smith- } \\
\text { Vaughan }\end{array}$ & [48] & + & + & + & + & - & - & + & + & + & Mod \\
\hline 2013, Stephen* & [42] & + & $?$ & + & + & - & - & + & + & + & Mod \\
\hline $\begin{array}{l}\text { 2015, Jervis- } \\
\text { Bardy }\end{array}$ & [57] & + & $?$ & + & + & + & + & + & + & + & Good \\
\hline 2015, Leach* & [47] & + & $?$ & + & + & - & - & + & + & + & Mod \\
\hline 2016, Leach* & [17] & + & $?$ & + & + & + & - & + & + & + & Mod \\
\hline
\end{tabular}

571 Data based on CASP-based risk of bias assessment. Assessment of bias pertained to the

572 microbiology data, and not to clinical data.

$573 *$ Indicates studies where microbiological outcomes were not the primary outcome. ? indicates

574 that this variable was unable to be assessed. 


\begin{tabular}{|c|c|c|c|c|c|c|c|c|c|c|c|c|c|c|c|c|c|c|c|c|c|c|}
\hline \multirow[b]{2}{*}{ 觮 } & \multirow[b]{2}{*}{ 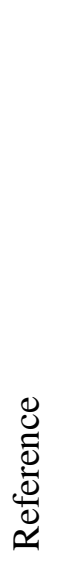 } & \multirow[b]{2}{*}{ 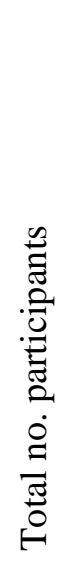 } & \multirow[b]{2}{*}{ 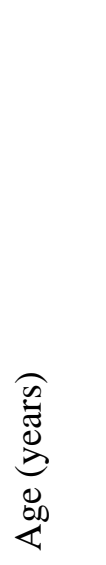 } & \multicolumn{4}{|c|}{ Study type } & \multicolumn{3}{|c|}{$\begin{array}{l}\text { Sample } \\
\text { site }\end{array}$} & \multicolumn{5}{|c|}{ Type of OM } & \multicolumn{7}{|c|}{ Analysis technique } \\
\hline & & & & 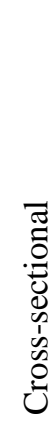 & 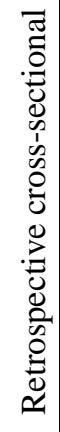 & 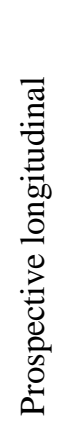 & 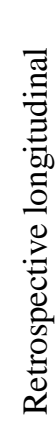 & 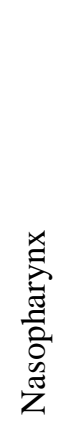 & $\begin{array}{l}0 \\
0 \\
\text { z } \\
0\end{array}$ & 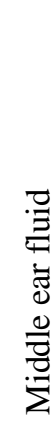 & $\sum_{0}^{2}$ & $\sum_{\substack{3 \\
\frac{3}{3}}}^{0}$ & $\sum_{0}^{11}$ & $\begin{array}{l}\sum_{0} \\
0 \\
\mathscr{U}^{2}\end{array}$ & 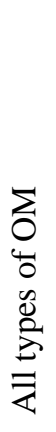 & 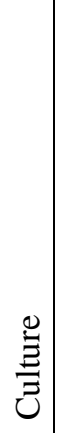 & 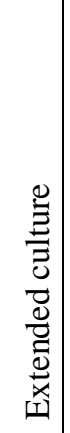 & 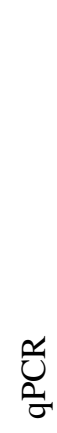 & 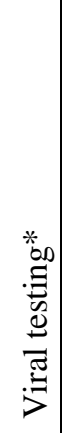 & 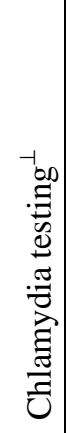 & 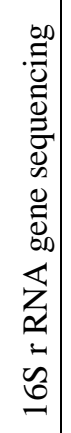 & $\begin{array}{l}\stackrel{0}{\Xi} \\
\stackrel{\Xi}{0} \\
.0 \\
0\end{array}$ \\
\hline \multicolumn{23}{|c|}{ Australian Indigenous } \\
\hline 1972, Stuart & [46] & 100 & $5-14$ & $\checkmark$ & & & & & & $\checkmark$ & & $\checkmark$ & & $\checkmark$ & & & $\checkmark$ & & & & & \\
\hline 1975, Stuart & [45] & 219 & $<2.5$ & & & $\checkmark$ & & & & $\checkmark$ & & $\checkmark$ & & & & & $\checkmark$ & & & & & \\
\hline $\begin{array}{l}1975, \\
\text { Copeman }\end{array}$ & [44] & 187 & $<15$ & $\checkmark$ & & & & & & $\checkmark$ & & $\checkmark$ & & & & $\checkmark$ & & & & & & \\
\hline $\begin{array}{l}1985, \\
\text { Dawson }\end{array}$ & [55] & 131 & $2-15$ & $\checkmark$ & & & & & & $\checkmark$ & & & $\checkmark$ & & & & $\checkmark$ & & & $\checkmark$ & & \\
\hline 1994, Leach & [21] & 41 & $<0.75$ & & & $\checkmark$ & & $\checkmark$ & & & & & & & $\checkmark$ & $\checkmark$ & & & & & & \\
\hline $\begin{array}{l}2003, \\
\text { Couzos }\end{array}$ & [40] & 147 & $<15$ & $\checkmark$ & & & & & & $\checkmark$ & & & & $\checkmark$ & & & $\checkmark$ & & & & & \\
\hline 2003, Stuart & [49] & 27 & $1-10$ & $\checkmark$ & & & & & & $\checkmark$ & & & $\checkmark$ & & & & $\checkmark$ & & & & & \\
\hline $\begin{array}{l}2005, \\
\text { Gibney ^ }\end{array}$ & [50] & 31 & $<8$ & & & $\checkmark$ & & $\checkmark$ & & $\checkmark$ & $\checkmark$ & $\checkmark$ & & & & $\checkmark$ & & & & & & \\
\hline $\begin{array}{l}\text { 2006, } \\
\text { Leach }^{\wedge}\end{array}$ & [51] & 21 & $<1.5$ & & & & $\checkmark$ & & & $\checkmark$ & & $\checkmark$ & & & & $\checkmark$ & & & & & & \\
\hline $\begin{array}{l}2007, \\
\text { Ashhurst- } \\
\text { Smith }\end{array}$ & [37] & 50 & $1-10$ & $\checkmark$ & & & & & & $\checkmark$ & & & $\checkmark$ & & & & $\checkmark$ & $\checkmark$ & & & & \\
\hline 2008, Leach & [43] & 97 & $1-15$ & $\checkmark$ & & & & & $\checkmark$ & $\checkmark$ & & & & $\checkmark$ & & & $\checkmark$ & & & & & \\
\hline 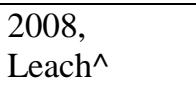 & [54] & 103 & NR & $\checkmark$ & & & & $\checkmark$ & & & & & $\checkmark$ & & & $\checkmark$ & & & & & & \\
\hline $\begin{array}{l}2009, \\
\text { Mackenzie }^{\wedge}\end{array}$ & [52] & 148 & $<2$ & & $\checkmark$ & & & & & $\checkmark$ & & $\checkmark$ & & & & $\checkmark$ & & & & & & \\
\hline $\begin{array}{l}2010, \text { Morris } \\
\pm\end{array}$ & [53] & 320 & $0.5-6$ & $\checkmark$ & & & & & $\checkmark$ & $\checkmark$ & & $\checkmark$ & & & & $\checkmark$ & & & & & & \\
\hline 2011, Binks^ & [35] & 115 & $<2$ & & & & $\checkmark$ & $\checkmark$ & & & $\checkmark$ & $\checkmark$ & $\checkmark$ & & & & & $\checkmark$ & & & & \\
\hline $\begin{array}{l}\text { 2012, } \\
\text { Marsh }^{ \pm}\end{array}$ & {$[56]$} & 27 & $0.5-4$ & & $\checkmark$ & & & $\checkmark$ & & $\checkmark$ & & $\checkmark$ & & & & & & $\checkmark$ & & & & \\
\hline $\begin{array}{l}\text { 2013, Smith- } \\
\text { Vaughan }\end{array}$ & [48] & 51 & $\begin{array}{c}0.25- \\
3.8\end{array}$ & & $\checkmark$ & & & $\checkmark$ & & $\checkmark$ & & $\checkmark$ & & & & $\checkmark$ & & $\checkmark$ & & & & \\
\hline $\begin{array}{l}2013, \\
\text { Stephen }\end{array}$ & [42] & 89 & $5-12$ & $\checkmark$ & & & & $\checkmark$ & & $\checkmark$ & & & & $\checkmark$ & & $\checkmark$ & & & & & & \\
\hline 2012, Sun & [36] & 66 & $<2$ & & & $\checkmark$ & & $\checkmark$ & & & & & & & $\checkmark$ & $\checkmark$ & & & & & & \\
\hline $\begin{array}{l}\text { 2015, Jervis- } \\
\text { Bardy }\end{array}$ & [57] & 11 & $3-10$ & $\checkmark$ & & & & $\checkmark$ & & $\checkmark$ & & & $\checkmark$ & & & & & & & & $\checkmark$ & \\
\hline 2015, Leach & [47] & 60 & $<6$ & $\checkmark$ & & & & & & $\checkmark$ & & $\checkmark$ & & $\checkmark$ & & $\checkmark$ & & & & & & \\
\hline 2016, Leach & [17] & 651 & $<6$ & $\checkmark$ & & & & & & $\checkmark$ & & $\checkmark$ & & $\checkmark$ & & $\checkmark$ & & & & & & \\
\hline
\end{tabular}




\begin{tabular}{|c|c|c|c|c|c|c|c|c|c|c|c|c|c|c|c|c|c|c|c|c|c|c|}
\hline \multirow[b]{2}{*}{$\underset{\vec{D}}{\stackrel{\vec{D}}{\Xi}}$} & \multirow[b]{2}{*}{ 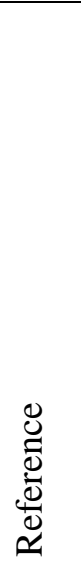 } & \multirow[b]{2}{*}{ 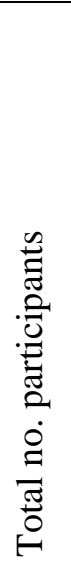 } & \multirow[b]{2}{*}{ 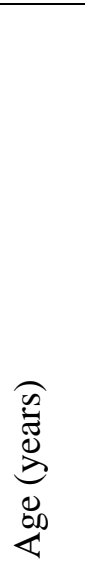 } & \multicolumn{4}{|c|}{ Study type } & \multicolumn{3}{|c|}{$\begin{array}{l}\text { Sample } \\
\text { site }\end{array}$} & \multicolumn{5}{|c|}{ Type of OM } & \multicolumn{7}{|c|}{ Analysis technique } \\
\hline & & & & 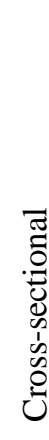 & 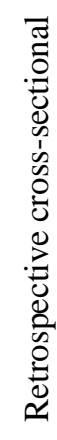 & 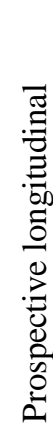 & 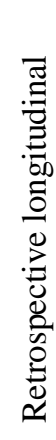 & 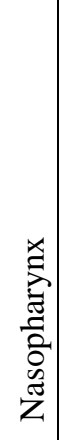 & $\begin{array}{l}0 \\
\dot{8} \\
z\end{array}$ & 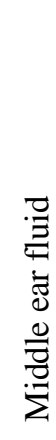 & \begin{tabular}{l}
$\Sigma$ \\
\hdashline \\
$\ll$
\end{tabular} & $\frac{\sum^{3}}{0}$ & $\sum_{0}^{\Perp}$ & $\begin{array}{l}\sum_{0} \\
\text { D }\end{array}$ & 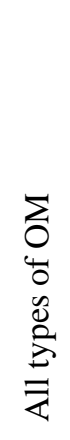 & $\stackrel{\Xi}{\Xi}$ & 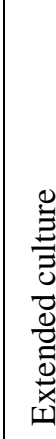 & 全 & 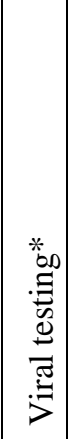 & 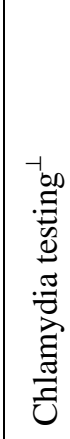 & 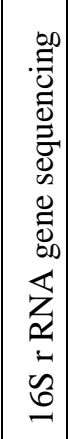 & (1) \\
\hline \multicolumn{23}{|c|}{ Greenlandic Inuit } \\
\hline $\begin{array}{l}1996, \\
\text { Homøe }\end{array}$ & {$[34]$} & 255 & $<10$ & $\checkmark$ & & & & $\checkmark$ & & $\checkmark$ & $\checkmark$ & $\checkmark$ & & & & & $\checkmark$ & & $\checkmark$ & $\checkmark$ & & \\
\hline $\begin{array}{l}2009, \\
\text { Homøe }\end{array}$ & [39] & 10 & $2-5$ & $\checkmark$ & & & & & & $\checkmark$ & & & $\checkmark$ & $\checkmark$ & & $\checkmark$ & & & & & & $\checkmark$ \\
\hline \multicolumn{23}{|c|}{ Alaskan Inuit } \\
\hline $\begin{array}{l}\text { 1999, } \\
\text { Parkinson }\end{array}$ & {$[38]$} & 128 & $<5$ & $\checkmark$ & & & & & & $\checkmark$ & & r & & & & & $\checkmark$ & & & & & \\
\hline
\end{tabular}

576 AOM, acute otitis media; AOMwP, acute otitis media with perforated tympanic membrane;

577 CSOM, chronic suppurative otitis media; OME, otitis media with effusion; No, number.

$578 *$ viral testing by qPCR [35] or PCR and immunofluorecent anti-body testing[11]; ${ }^{\perp}$ Chlamydia

579 spp. testing by qPCR [11] and immunofluorecent anti-body testing[55]; ${ }^{\circ}$ biofilm testing by

580 immersion microscopy Gram-staining, PNA-FISH (for S. aureus, coagulase negative

581 staphylococcus, Escherichia coli, and eubacterial probe) and confocal laser scanning

582 microscopy; ${ }^{\wedge}$ Overlapping participants; ${ }^{ \pm}$Overlapping participants.

583 Note: For the longitudinal studies, data included in this review was per specimen, not per child. 


\section{Figure 1: Literature search and selection}

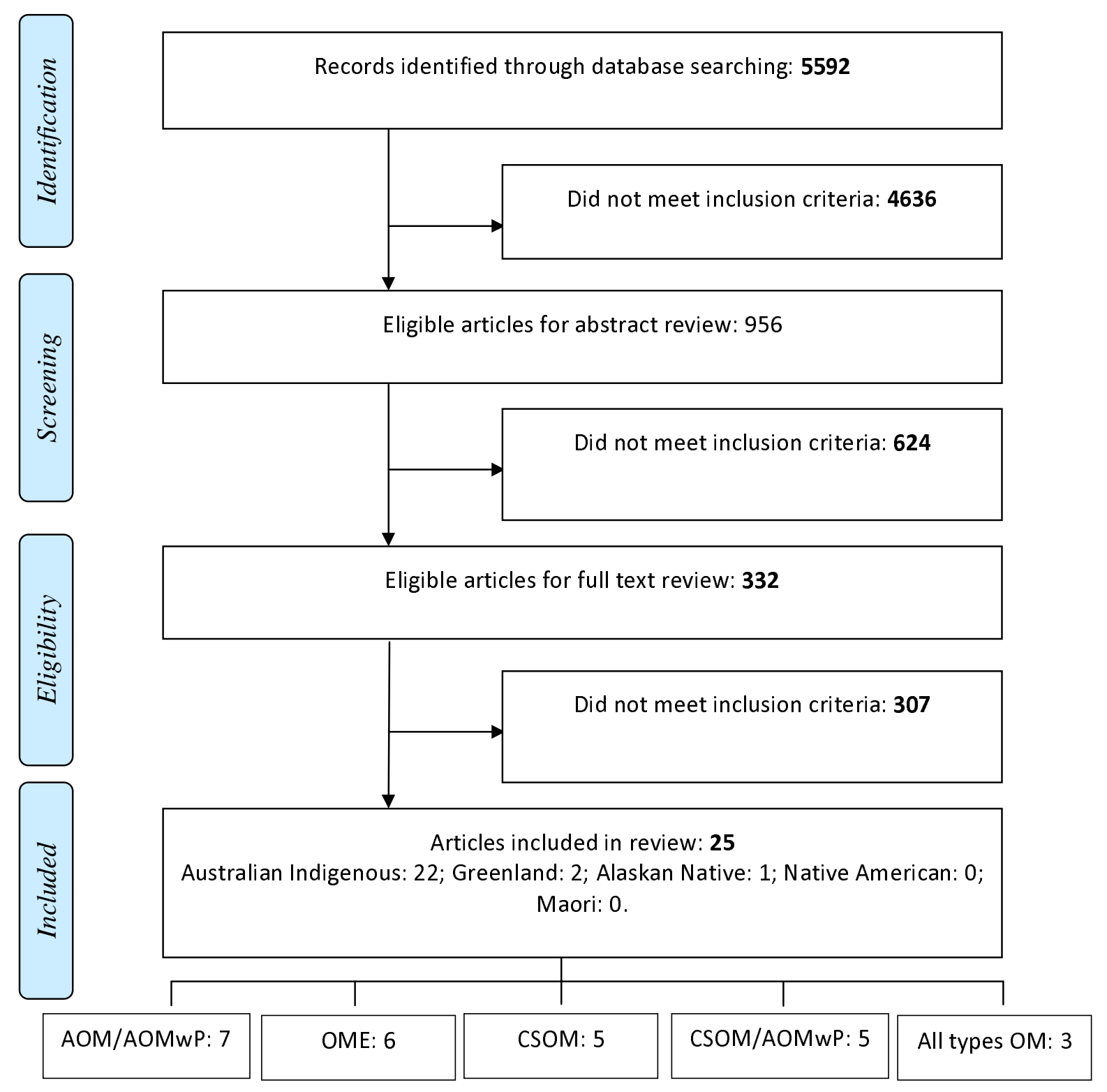




\section{Figure 2: Bacteriology in relation to acute otitis media}

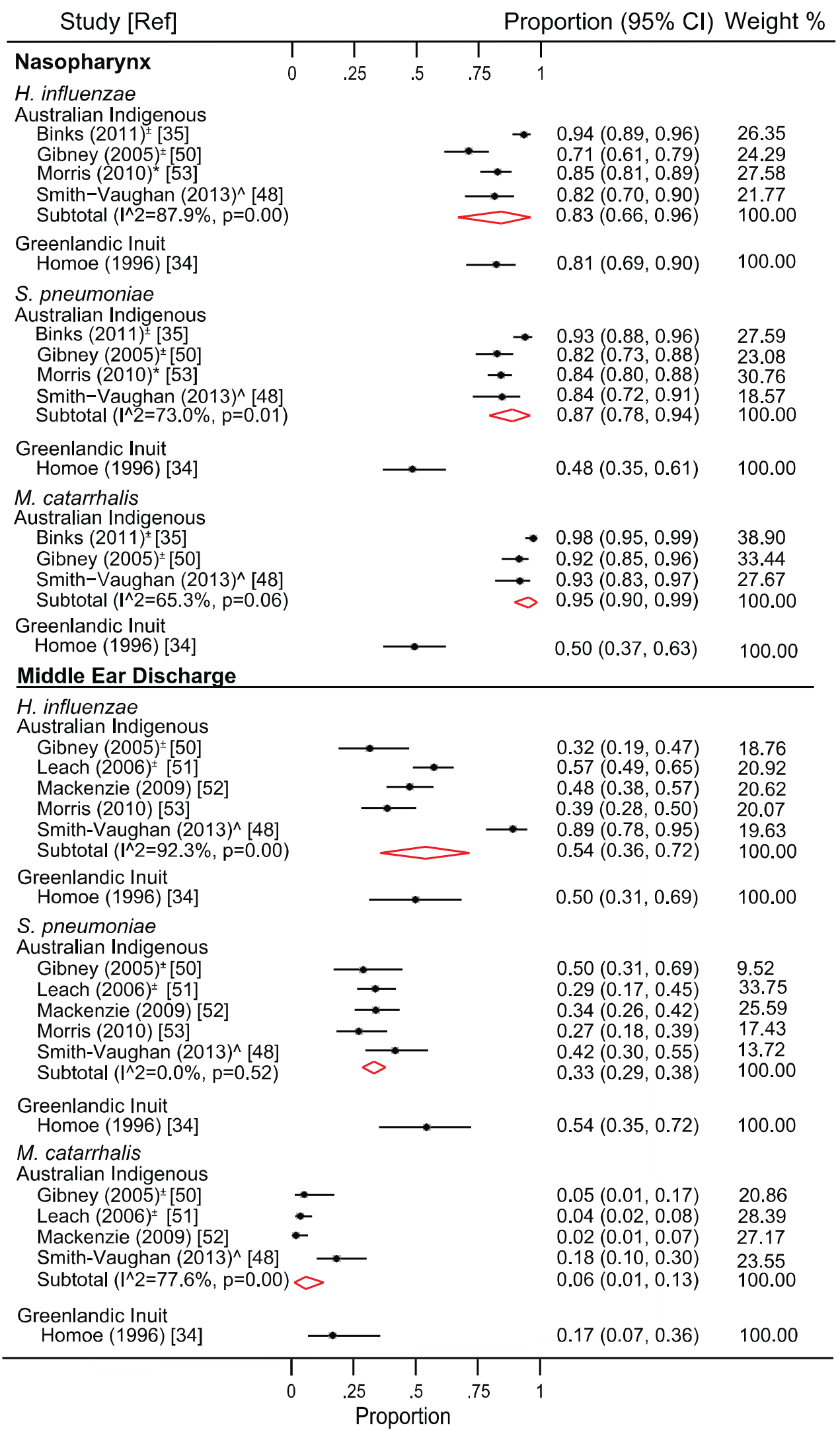

Note: Binks et al, 2011 combines AOM and AOMwP. * nasal swabs. ^ PCR analysis; U/C, unable to calculate. 


\section{Figure 3: Bacteriology in relation to otitis media with effusion}

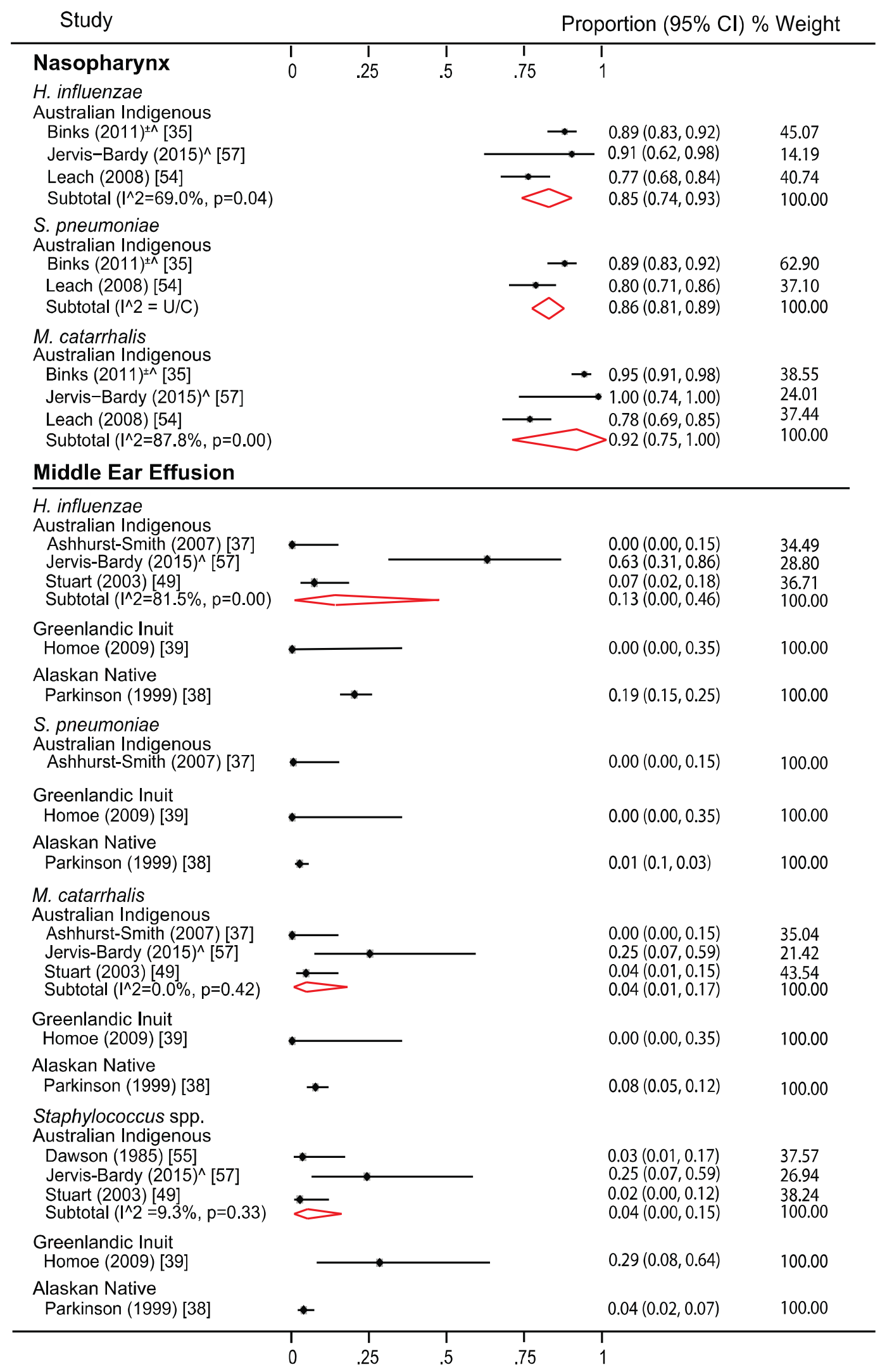

Note: ^ PCR/ next generation sequencing; U/C, unable to calculate. 
Figure 4: Bacteriology in relation to chronic suppurative otitis media

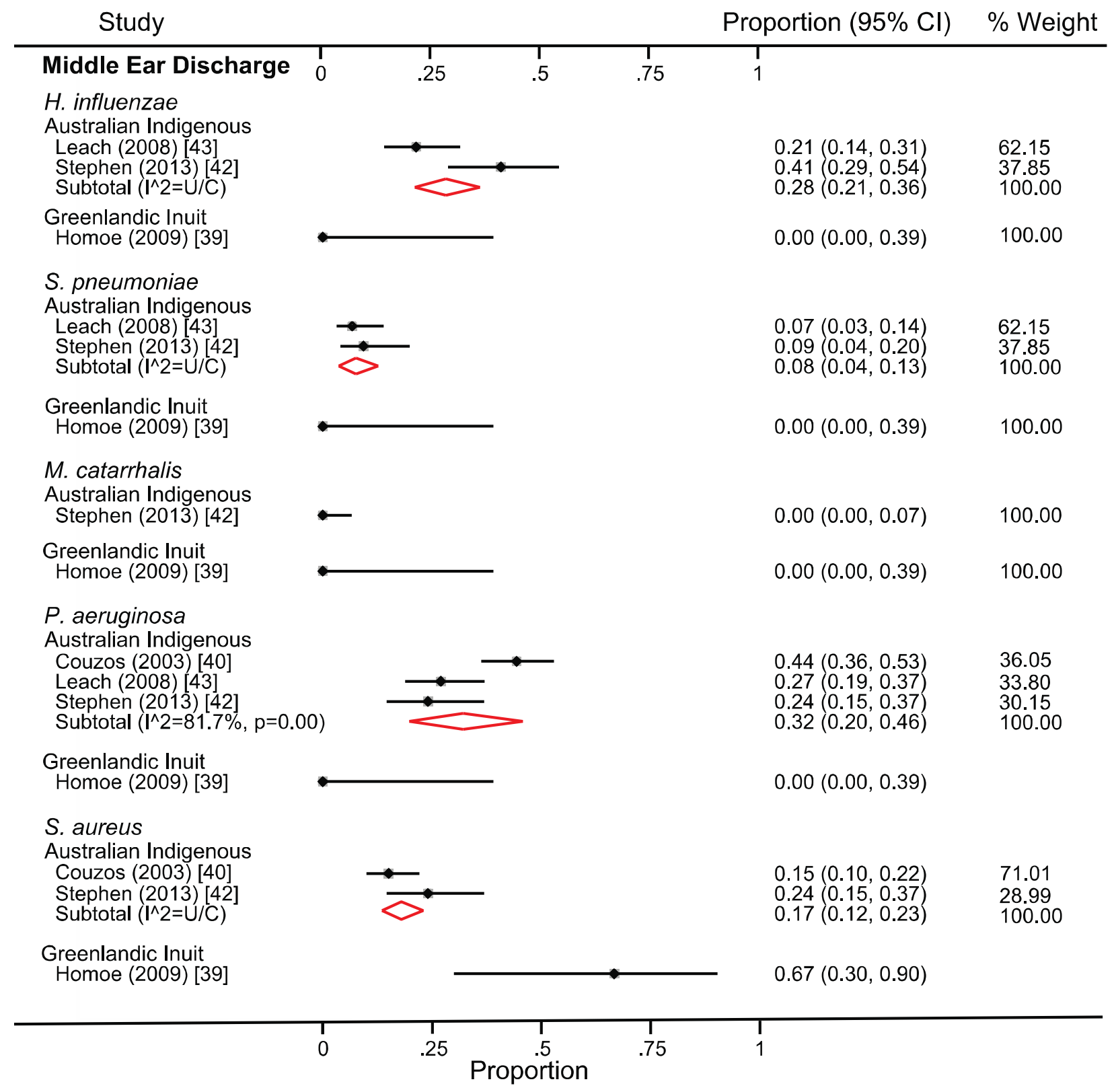

Note: U/C, unable to calculate. 
Figure 5: Recommendations for future research of OM microbiology in indigenous children

- Exploration of entire URT microbiota in indigenous populations. Understanding the entire microbiota can explore the hypothesis of dysbiosis in these populations. This can be achieved using either:

○ Metagenomic shot gun sequencing if techniques to remove human DNA advance;

○ 16S rRNA gene sequencing with adjunct qPCR; or

- Extended culture with MALDI-TOF and adjunct qPCR - creates microbial resources for further studies, such as bacterial interference.

- Inclusion of healthy indigenous peers in microbiota studies to increase knowledge of the 'healthy' URT microbiota and identification of possible 'protective' microbes

- Inclusion of indigenous participants from diverse geographical, climactic and socioeconomic backgrounds to facilitate generalisation of results.

- Internationally standardised OM diagnostic criteria are needed to enable comparison of data amongst studies from different populations.

- Healthy indigenous control groups

- New therapies to prevent/ reduce colonisation with otopathogens in indigenous populations, including microbiome/ probiotic therapies. 